\title{
Nano-ZnS thin films for solar cell
}

\author{
M. A. Jafarov, E. F. Nasirov, S. A. Jahangirova, R. Jafarli \\ Baku State University, Baku, Azerbaijan \\ maarif.jafarov@mail.ru
}

PACS 71.55.Gs, 78.20.e, 85.30.z, 85.60.Dw DOI 10.17586/2220-8054-2015-6-5-644-649

In this paper, we report the effect of deposition time on the properties of zinc sulfide thin films. The $\mathrm{ZnS}$ thin films have been grown on amorphous glass substrates and at various deposition periods ranging from 30 to 120 min by chemical bath deposition technique. Other parameters, such as reactant concentration, solution $\mathrm{pH}$, and bath temperature were kept constant for the all depositions. Morphological characterizations of the surface were studied using the atomic force microscopy (AFM). The AFM images confirmed that the grain size of ZnS increased with increased deposition time. Average diameter of nanoparticles was between 60 and $90 \mathrm{~nm}$, while the roughness ranged from 6 to $11 \mathrm{~nm}$. Optical properties, which were determined from UV-VIS spectrophotometry, were obtained by analyzing the measured absorbance and transmittance spectrum. The zinc sulfide thin films show high transmittance in the visible region and the $\mathrm{ZnS}$ band gap value was estimated to be in range of $3.99-4.05 \mathrm{eV}$.

Keywords: zinc sulfide, nanoparticles, chemical bath deposition.

Received: 10 June 2015

Revised: 25 June 2015

\section{Introduction}

Zinc sulfide $(\mathrm{ZnS})$ is an important II - VI semiconducting material with a wide direct band gap of $3.65 \mathrm{eV}$ in the bulk [1-5]. This new class of materials has not only provided many unique opportunities but also exhibited novel optical and transport properties, which are potentially useful for technological applications. These materials have potential application in optoelectronic devices, such as blue light emitting diodes, electroluminescent devices and photovoltaic cells [1-3] and more recently as $n$-type nonreflecting window materials in heterojunction solar cells. The efficiency of heterojunction solar cells depend largely on the interfacial properties between absorber and buffer layers. The wider bandgap of $\mathrm{ZnS}$ enables high energy incident photons to reach the window-absorber junction, enhancing the blue response of the photovoltaic cells and thus contributes to a better cell performance [4]. Recently, efficiency enhancement of solar cells has become more important due to the need for environmentally benign renewable energy sources. To improve the light harvesting in solar cells, it is crucial to minimize undesirable Fresnel surface reflection losses at the interface between air and the top layer of solar cells over the entire range of the solar spectrum.

Zinc sulfide has found wide use as a thin film coating in the optical and microelectronic industries, having a high refractive index $(2 \times 25$ at $632 \mathrm{~nm})$, high effective dielectric constant $(9$ at $1 \mathrm{MHz})$ and wide wavelength pass band $(0 \times 4-13 \mathrm{~mm})$ [5]. There have been various studies on the bulk and thin film characteristics of $\mathrm{ZnS}$, including optical and electrical properties [6]. The optical properties of the prepared film depend strongly on the manufacturing technique. Two of the most important optical properties; refractive index and the extinction coefficient are generally called optical constants. The amount of light which is transmitted through thin film material depends on the amount of the reflection and absorption that takes place along the light path [7]. 
Many growth techniques have been reported for the preparation of $\mathrm{ZnS}$ thin films, such as sputtering, pulsed-laser deposition, metal organic chemical vapor deposition, electron beam evaporation, photochemical deposition, thermal evaporation, sol-gel processing, co-precipitation and chemical bath deposition [8]. These doped $\mathrm{ZnS}$ semiconductor materials have a wide range of applications in electroluminescence devices, phosphors, light emitting displays, and optical sensors. Doped nanoparticles with dimensions below that of the Bohr diameter exhibit interesting optoelectronic properties due to quantum size effect and are potential candidates for a variety of applications. The characteristics and concentrations of dopants are responsible for particular luminescence emission and efficiency of semiconductor nanoparticles [9]. Hence, investigation of the role that dopant concentration has on doped semiconductor nanoparticle optical properties of is very important from the viewpoints of basic and applied physics [10]. Recently, 2D nanostructure P-N junctions have attracted a great deal of attention for their potential application in photovoltaic devices. Zinc sulfide $(\mathrm{ZnS})$ was one of the first semiconductors discovered and is also an important semiconductor material with direct wide band gaps for cubic and hexagonal phases of 3.72 and $3.77 \mathrm{eV}$, respectively $[9,11]$. It has a high absorption coefficient in the visible range of the optical spectrum and reasonably good electrical properties. This property makes $\mathrm{ZnS}$ a very attractive as an absorber in heterojunction thin-film solar cells. Among various other methods, the chemical bath deposition - CBD that we use in present case is a well known deposition process for some chalcogenides, it is well known as prevented low temperature aqueous technique for deposition large area of semiconductor thin films and has gained renewed interest due to the good quality and high purity of the deposited films. CBD of $\mathrm{ZnS}$ is a highly reproducible and controllable technique, thus it is considered the simplest and the most economical one. CBD results good deposits on suitable substrates by controlled precipitation of the compound from the solution.

\section{Experiment}

The basic principle of the CBD technique consists in the controlled generation of the metal and chalcogenide ions in an alkaline medium and their ion by ion deposition on the substrate in order to form a film. In our CBD experiment, the deposition of $\mathrm{ZnS}$ film was based on the reaction between zinc sulfate $\left(\mathrm{ZnSO}_{4}\right)$ as a source of $\mathrm{Zn}^{2+}$, Thiourea $\left(\mathrm{SC}\left(\mathrm{NH}_{2}\right)_{2}\right)$ as a source of $\mathrm{S}^{2-}$ and ammonia $\left(\mathrm{NH}_{4} \mathrm{OH}\right)$ that used as complexing agent to vary the $\mathrm{pH}$ of the reaction bath and to control the $\mathrm{Zn}^{2+}$ concentration.

$\mathrm{ZnS}$ films were deposited on commercial glass substrates $(75 \mathrm{~mm} \times 25 \mathrm{~mm} \times 1 \mathrm{~mm})$ by chemical bath deposition technique. Prior to deposition, the substrate was degreased in ethanol for $10 \mathrm{~min}$, followed by ultrasonic cleaning with doubly-distilled water for another $10 \mathrm{~min}$, rinsed in de-ionized water and finally air dried in. In a typical deposition set up of $\mathrm{ZnS}$, the CBD was achieved by mixing $10 \mathrm{ml}$ of $0.025 \mathrm{M} \mathrm{ZnSO}_{4}$ solution, $10 \mathrm{ml}$ of $0.27 \mathrm{M}$ $\mathrm{SC}\left(\mathrm{NH}_{2}\right)_{2}$ solution, and $2.9 \mathrm{M} \mathrm{NH}_{4} \mathrm{OH}$ solution. Firstly, ammonia solution was added slowly to the required quantity of zinc sulfate, after stirring for several minutes the solution becomes colorless and homogeneous, thereafter, a thiourea solution was added under stirring. Lastly, the reaction solution was placed in $50 \mathrm{ml}$ beaker (sealed with a Teflon tape) into the water bath pot. The glass substrates were then immersed vertically inside this beaker and supported against the wall of the beaker without disturbing it. The deposited film was carried out water bath temperature at $T_{b}=70{ }^{\circ} \mathrm{C}$, for different durations, $(30,60,90,120 \mathrm{~min})$ that were selected to study the characteristics of $\mathrm{ZnS}$ thin films. After complete film deposition, each sample was removed from the beaker and was cleaned with de-ionized water to remove the white, loosely, adherent powders precipitate in the solution during the deposition. The structural properties of $\mathrm{ZnS}$ films are studied by using X-ray diffractometer (XRD) using $\mathrm{Cu}-\mathrm{K} \alpha$ radiation with 
wavelength $1.5418 \AA$. Surface morphology was examined by Scanning Electron Microscope (SEM). The atomic force microscopy (AFM) was used to investigate the surface morphology and surface roughness. AFM imaging is performed on the Nanosurf system (easyScan2) operating in a tapping mode in air at room temperature. The optical transmission and absorption studies of the deposited $\mathrm{ZnS}$ thin films were carried out with a UV-VIS spectrophotometer (Varian carry 5000). Absorption coefficient corrected for scattering contribution versus wavelength. The error bars represent the standard deviation.

\section{Results and discussion}

AFM allows us to obtain microscopic information on the surface structure and to plot topographies representing the surface relief. In this work, we have used this technique to visualize the surface relief, specify the growth, and determine the contribution of the deposition time $\left(t_{d}\right)$ to the quality of the film. AFM images of the surface morphology recorded on samples of the ZnS-NPs thin films deposited with different deposition times for $\left(t_{d}=10,40 \mathrm{~min}\right)$ are shown in Fig. 1. In all cases the prepared thin films have good quality, uniform morphology and covered the entire substrate surface. We can expect this result due to the increase of the film thickness with the deposition time.
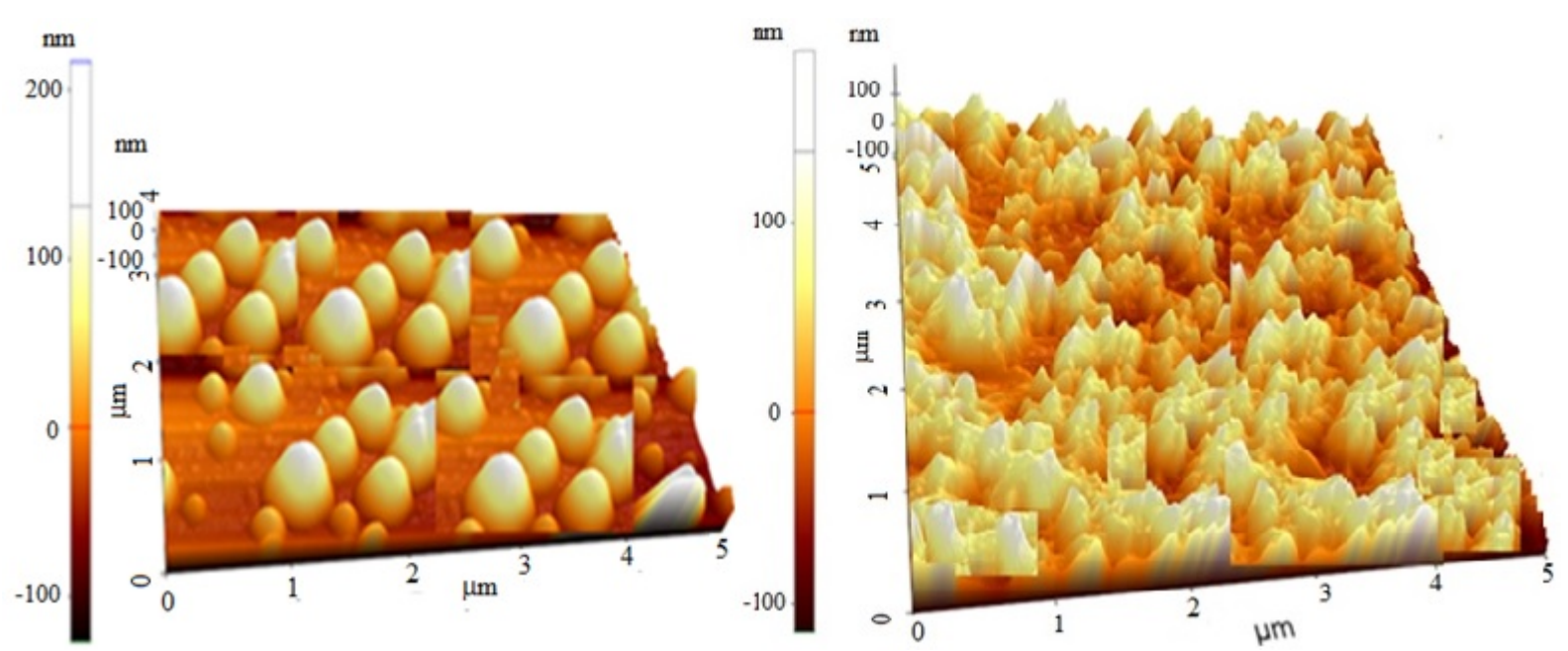

FIG. 1. AFM images of the surface morphology recorded on samples of the $\mathrm{ZnS}-\mathrm{NPs}$ thin films deposited with different deposition time periods for $(t, \min$ : $10,40)$

The same relation between the mean height of $\mathrm{ZnS}-\mathrm{NPs}$ and the deposition time has been observed. This can be seen in the increasing roughness with increased deposition time and that because new bigger domed grains are formed, the layer is more complete and has fewer voids. It is important to note that these obtained values are averaged and there is a statistical variation associated with them, which depends on the location of the measurement that is performed on the samples. To minimize these errors, we performed many measurements of each parameter at several different locations on the surface of samples.

The optical properties evolution for the prepared $\mathrm{ZnS}$ thin films was determined after obtaining the absorbance and transmittance spectrum with a UV-VIS spectrophotometer. UVVIS transmittance spectra of ZnS-NPs formed at bath or reaction temperature of $70{ }^{\circ} \mathrm{C}$ with different reaction times $(30,60 \mathrm{~min})$ are given in Fig. 2. Transmittance was recorded from $300-$ $800 \mathrm{~nm}$. The transmission of the zinc sulfide thin films decreases with increased deposition 
times. The average transmittance of these films is calculated to be $99 \%, 97.5 \%, 96 \%$, and $95 \%$, respectively. These results are related to the increased deposition time; as a result of the voids in the $\mathrm{ZnS}$ thin layer filling up with new $\mathrm{ZnS}$ grains, film thickness is increased, thus reducing the transmittance. In general, the layers produced in this work exhibit high transmittance, which depends on low film thickness. In addition, the decreased transmittance can be linked with agglomeration and increased grain size, indicating its high surface roughness. The $\mathrm{ZnS}$ thin films have a steep optical absorption feature, indicating good homogeneity in the shape and size of the ZnS-NPs and low defect density.

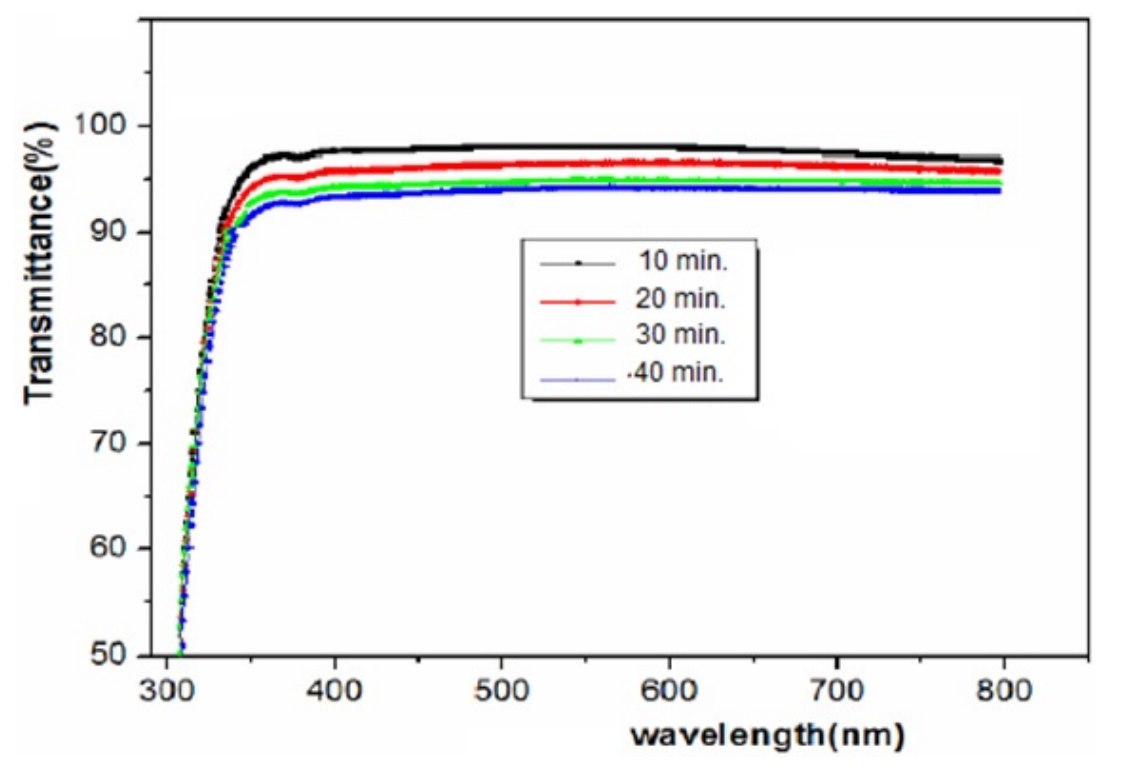

FIG. 2. Transmittance spectra of $\mathrm{ZnS}$ thin film at different deposition times (10, 20, 30, $40 \mathrm{~min})$

Figure 3 shows the absorption coefficient a as a function of photon energy; at low photon energies, a decreases the probability of electrical transfer between the valance and conduction bands, making it very rare and conversely, will increase at the absorbance edge, towards high energy photons.

Using the last data, the band gap energy of $\mathrm{ZnS}$ thin film was estimated by plotting, as in Fig. 4. The linear nature of the plot indicates that $\mathrm{ZnS}$ is a direct band gap material. The films' optical band gaps can be evaluated by extrapolating the straight portion to the axis.

In this work, the band gap energy $\left(E_{g}\right)$ was determined to be in the range of $4.05-$ $3.6 \mathrm{eV}$ for the $\mathrm{ZnS}$ films with different deposition times, from 10 to $40 \mathrm{~min}$, respectively which closely agree with the values reported for $\mathrm{ZnS}$ thin films obtained by CBD. The band gap values of the as deposited $\mathrm{ZnS}$ films are somewhat larger than the typical band gap for bulk $\mathrm{ZnS}(\sim 3.6 \mathrm{eV})$ at $300 \mathrm{~K}$. In Fig. 4, we have reported the variation of band gap energy and thickness of thin film $\mathrm{ZnS}-\mathrm{NPs}$ as a function of deposition time. We note a reduction in the gap with the deposition time this due to the quantum size effect as expected for the nanoparticles nature of the films. In fact, it is observed that the band gap energy values of $\mathrm{ZnS}$ thin layers decrease slightly from 4.05 to $3.99 \mathrm{eV}$ with increased deposition time. There was good agreement between the structural and optical properties and calculated band energy gap. The thickness of $\mathrm{ZnS}$ thin film slightly increased from 65 to $90 \mathrm{~nm}$ when the deposition time was increased from 10 to $40 \mathrm{~min}$. This may be due to processes of heterogeneous and homogenous precipitation, which increase with time, leading to higher $\mathrm{ZnS}$ thin film growth rates. 


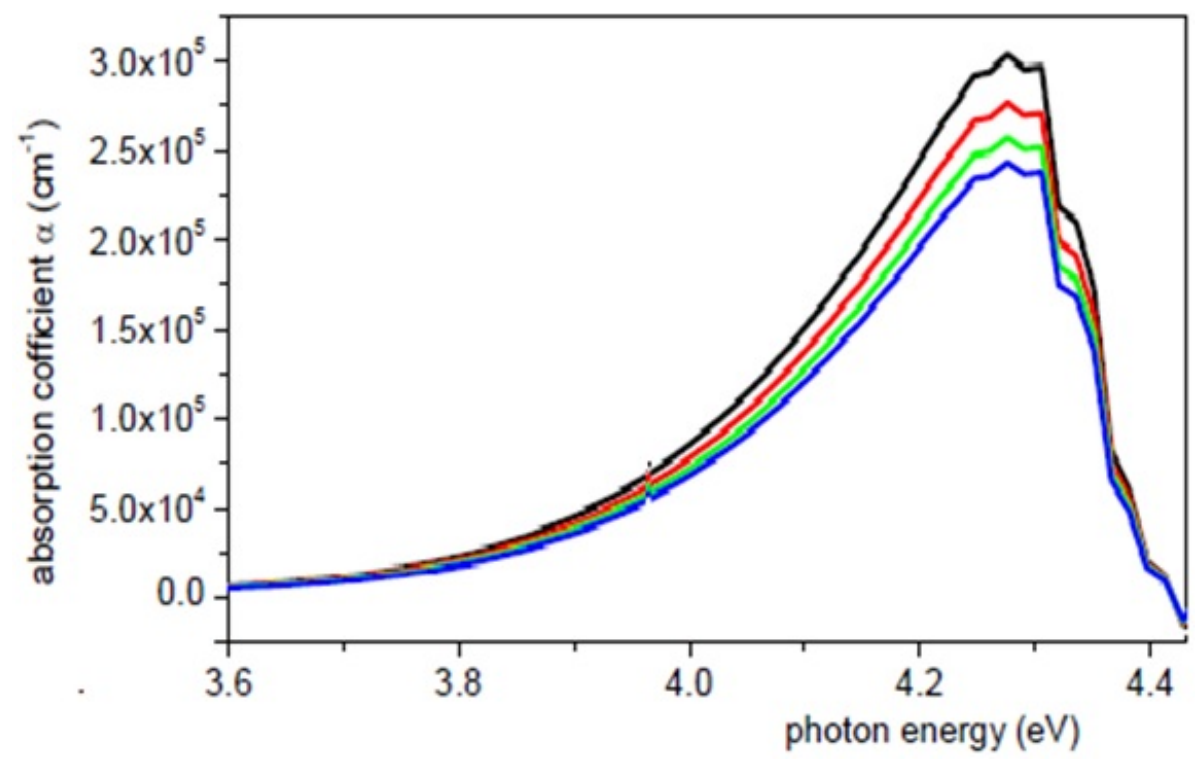

FIG. 3. Absorption coefficient versus photon energy for $\mathrm{ZnS}$ thin films deposited at different times $(10,20,30,40 \mathrm{~min})$
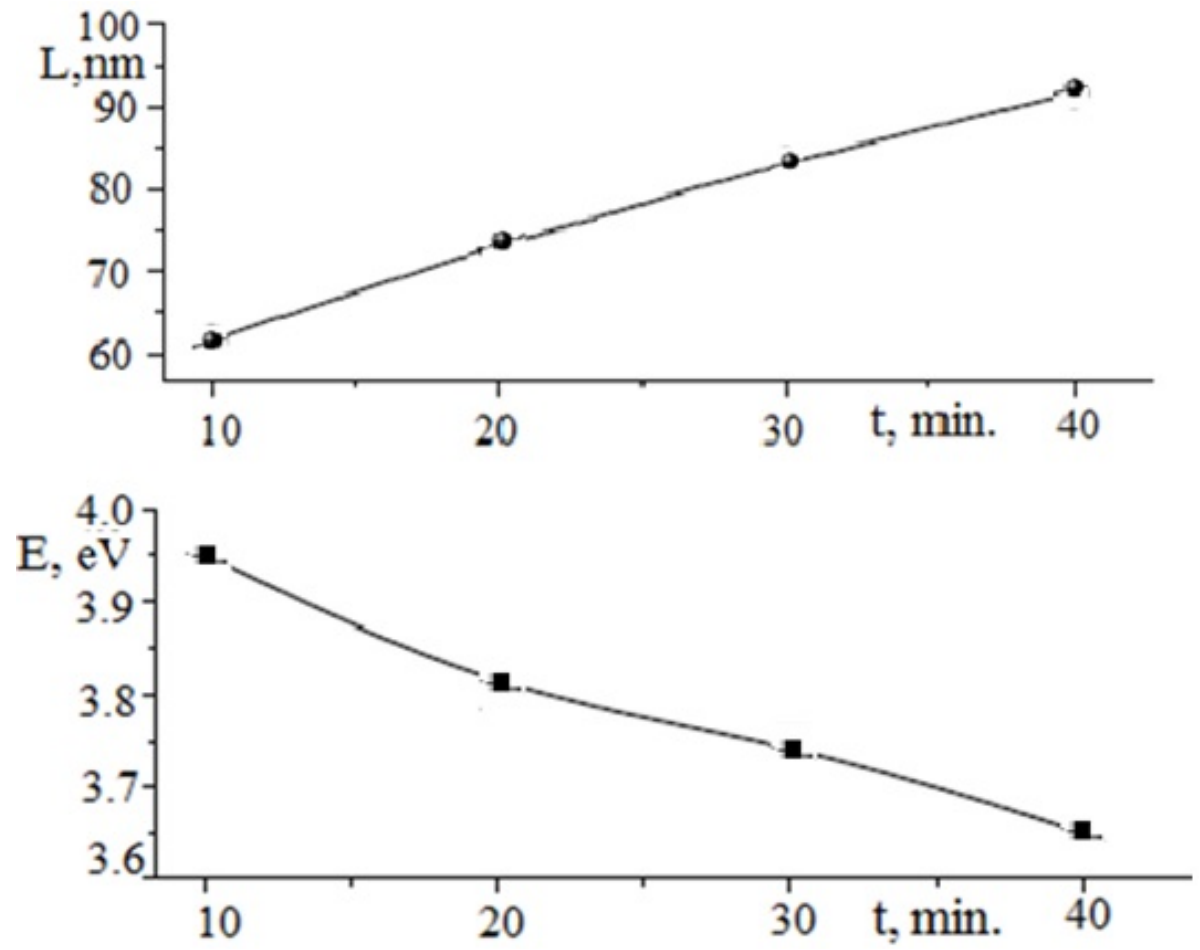

FIG. 4. Variation of $\mathrm{ZnS}$ thin films thickness and band gap with deposition times at fixed bath temperature $T_{b}=70{ }^{\circ} \mathrm{C}$ 


\section{Conclusion}

We have successfully used chemical bath deposition to obtain $\mathrm{ZnS} /$ glass thin films. AFM showed that the films were of good quality and have uniform distribution of ZnS-NPs over the glass substrate. Images revealed that the particle size increased with increasing of deposition time, the average diameter was $180 \mathrm{~nm}$. The high transparency of the films, over $90 \%$, was investigated using a UV-VIS spectrophotometer. The optical direct band gap energy was calculated to be between $3.99-4.05 \mathrm{eV}$ for the $\mathrm{ZnS}$ films with different thicknesses. Structural and optical properties were in agreement with each other. From these studies, we are able to optimize the process in order to produce the antireflection layer of $\mathrm{ZnS}$ suitable for optical window in solar cells.

\section{References}

[1] T. Ben Nasr, N. Kamoun, M. Kanzari, R. Bennaceur. Effect of $\mathrm{pH}$ on the properties of $\mathrm{ZnS}$ thin films grown by chemical bath deposition. Thin Solid Films, 2006, 500, P. 4-8.

[2] A.U. Ubale, D.K. Kulkarni. Preparation and study of thickness dependent electrical Characteristics of zinc sulfide thin films. Bull. Mater. Sci., 2005, 28 (1), P. 43-47.

[3] N.M. Saeed. Structural and Optical Properties of ZnS Thin Films Prepared by Spray Pyrolysis Technique. Journal of Al-Nahrain University, 2011, 14 (2), P. 86-92.

[4] R. Sharma, B.P. Chandra, D.P. Bisen. Optical properties of ZnS: Mn nanoparticles prepared by chemical bath routs. Chalcogenide Letters, 2009, 6 (8), P. 339-342.

[5] J. Hasanzadeh, A. Taherkhani, M. Ghorbani. Luminescence and Structural Properties of ZnS: Cu Nanocrystals Prepared Using a Wet Chemical Technique. Chinese journal of physics, 2013, 51 (3), P. 540-550.

[6] X. Wu, F. Lai, et al. Effects of substrate temperature and annealing on the structure and optical properties of ZnS film. Proc. of SPIE, 2007, 6722, 67222L(1-5).

[7] K. Jayanthi, S. Chawla, H. Chander, D. Haranath. Structural, optical and photoluminescence properties of $\mathrm{ZnS}$ : $\mathrm{Cu}$ nanoparticle thin films as a function of dopant concentration and quantum confinement effect. Cryst. Res. Technol., 2007, 42 (10), P. 976-982.

[8] L.-W. Ji, Y.-J. Hsiao, et al. Annealing effect and photovoltaic properties of nano-ZnS/textured p-Si heterojunction. Nanoscale Research Letters, 2013, 8 (470), P. 1-6.

[9] S.P. Patel, J.C. Pivin, et al. Grain growth and structural transformation in ZnS nanocrystalline thin films. Vacuum, 2010, 85, P. 307-311.

[10] M. Ashrat, M. Mehmood, A. Qayyum. Influence of source-to-substrate distance on the properties of ZnS films grown by close-space sublimation. Semiconductors, 2012, 46 (10), P. 1326-1330.

[11] H. Abdullah, N. Saadah, S. Shaari. Effect of Deposition Time on ZnS Thin Films Properties by Chemical Bath Deposition (CBD) Techinique. World Applied Sciences Journal, 2012, 19 (8), P. 1087-1091. 